\title{
Multiplicando Saberes:Ampliando os Cuidados em Saúde Bucal. Relato de Experiência de um Projeto de Extensão Universitária
}

\section{Multiplying Knowledge: Extending Oral Health Care. Experience Report of a University Extension Project}

\section{Multiplicar el conocimiento: extender la atención de la salud bucal. Informe de experiencia de un proyecto de extensión universitaria}

Filipe de Souza Nunes

Vanessa Viana dos Santos

Paulo Ricardo Saquete Martins Filho ${ }^{1}$

RESUMO: A extensão universitária é uma atividade que engrandece a vivência acadêmica, implementando melhorias na qualidade de saúde bucal da comunidade. Esse escopo teve como objetivo mostrar o desenvolvimento de práticas educativas e promoção de saúde no projeto de Extensão Multiplicando Saberes o qual é vinculado ao Programa de Pós-Graduação em Odontologia (PRODONTO) da UFS. A ação de extensão envolveu atividades educativas voltadas à cidadania e promoção de saúde bucal, com orientação e avaliação odontológica a crianças e adolescentes com deficiência e seus cuidadores, professores e orientadores educacionais. Participaram 162 crianças e adolescentes com deficiência das quais $22 \mathrm{com}$ paralisia cerebral, $36 \mathrm{com}$ transtorno do espectro autista, 13 com síndrome de Down e 91 com retardo mental. Dos 44 cuidadores participantes, $92 \%$ eram mulheres. A faixa etária da maioria foi de 30 a 40 anos (54.5\%), casados (38.6\%), tendo 2 a 3 filhos (63.6\%),e ensino médio completo (72.7\%) e são cuidadores integrais $79.5 \%$. Cerca de 68.2\% não possuem renda formal. Dos 4 educadores participantes, 3 são do gênero feminino e apresentam ensino médio completo. Foi possível observar a materialização de noções básicas de saúde, o autocuidado e a capacitação de cuidadores, elevados à condição de agentes ativos e multiplicadores das ações de promoção de saúde em nível individual e coletivo.

1Programa de Pós-Graduação em Odontologia, departamento de Odontologia - Universidade Federal de Sergipe 
Descritores: educação em saúde bucal; extensão; comunidade.

ABSTRACT: Introduction: University extension is an activity that enhances the academic experience, implementing improvements in the oral health quality of the community. Objective: This scope aimed to show the development of educational practices and health promotion in the Extension Multiplicando Saberes project, which is linked to the Postgraduate Program in Dentistry (PRODONTO) of UFS. Material and Method: The extension action involved educational activities focused on citizenship and oral health promotion, with orientation and dental assessment to children and adolescents with disabilities and their caregivers, teachers and educational counselors. RESULTS: A total of 162 children and adolescents with a disability participated, 22 of them with cerebral palsy, 36 with autism spectrum disorder, 13 with Down syndrome and 91 with mental retardation. Of the 44 participating caregivers, 92\% were women. The majority age group was between 30 and 40 years old (54.5\%), married (38.6\%), having 2 to 3 children (63.6\%), and complete secondary education (72.7\%). About $68.2 \%$ have no formal income. Of the 4 participating educators, 3 are female and have completed high school. Conclusion: It was possible to observe the materialization of basic concepts of health, self-care and the training of caregivers, elevated to the condition of active agents and multipliers of actions of health promotion at individual and collective level.

Descriptors: oral health education; extension; community.

RESUMEN: La extensión universitaria es una actividad que mejora la experiencia académica, implementando mejoras en la calidad de la salud oral de la comunidad. Este alcance tuvo como objetivo mostrar el desarrollo de las prácticas educativas y la promoción de la salud en el proyecto de Extensión del Conocimiento Multiplicador que está vinculado al Programa de Postgrado en Odontología de UFS (PRODONTO). La acción de extensión incluyó actividades educativas dirigidas a la ciudadanía y la promoción de la salud bucal, con orientación y evaluación dental para niños y adolescentes con discapacidades y sus cuidadores, maestros y consejeros educativos. Participaron 162 niños y adolescentes con discapacidad, 22 con parálisis cerebral, 36 con trastorno del espectro autista, 13 con síndrome de Down y 91 con retraso mental. De los 44 cuidadores participantes, el 92\% eran mujeres. El grupo de edad mayoritario fue de 30 a 40 años (54.5\%), casados (38.6\%), con 2 a 3 hijos (63.6\%) y completar la escuela secundaria (72.7\%) y cuidadores completos $79.5 \%$. Alrededor del 68.2\% no tiene ingresos formales. De los 4 educadores participantes, 3 son mujeres y han completado la escuela secundaria. Fue posible observar la materialización de los conceptos básicos de salud, autocuidado y capacitación de cuidadores, elevada a la condición de agentes activos y multiplicadores de acciones de promoción de la salud a nivel individual y colectivo.

Palabras clave: educación en salud bucal; extensión comunidad.

\section{INTRODUÇÃO}

A extensão universitária é o processo educativo, cultural e científico que articula o ensino e Tempus, actas de saúde colet, Brasília, 13(3), 183-190, set, 2019. Epub Jul/2020 ISSN 1982-8829 
a pesquisa de forma indissociável e viabiliza a relação transformadora entre a Universidade e a sociedade. Através da extensão, vários setores da sociedade se vinculam com a Universidade, onde a mesma oferece à comunidade conhecimentos e assistência, e recebe de volta informações essenciais sobre valores e cultura que serão replicados de forma válida e globalizada para toda a comunidade.

A extensão tem sido considerada uma atividade capaz de imprimir novos rumos à formação acadêmica, constituindo-se instrumento indispensável de aprendizagem e de formação profissional e pessoal ${ }^{1}$. Entretanto, no cenário da pós-graduação brasileira, a extensão, por muitas vezes, tem tomado um lugar secundário no planejamento das atividades, contribuindo para práticas de pesquisa e ensino dissociadas da realidade ${ }^{2}$. Portanto, o reconhecimento da indissociabilidade entre ensino, pesquisa e extensão "deveria promover uma nítida articulação entre aquelas três atividades acadêmicas, em que o professor da pós-graduação, como orientador, tem papel de motivador, supervisor e avaliador de todo o processo de pesquisa em que se encontram seus alunos, ajudandoos a enxergar nessa atividade uma ética balizada pelo bem-estar das comunidades envolvidas nas pesquisas" 2 .

Apesar da existência de assimetrias regionais, a pós-graduação no Brasil tem se expandido nos últimos anos, trazendo à tona discussões quanto à sua responsabilidade social. Recentemente, com a criação do ítem Inserção Social como quesito de avaliação dos cursos de pós-graduação pela CAPES, houve um explícito reconhecimento de que a produção de conhecimento e de formação de quadros deve se articular com a dinâmica social, alcançando-a de modo mais direto e imediato ${ }^{3}$.

O Programa de Pós-Graduação em Odontologia da Universidade Federal de Sergipe, criado em 2012 e preocupado com sua responsabilidade social e com o impacto de seus produtos no desenvolvimento socioeconômico da região, tem voltado seus esforços na aplicação sistêmica de atividades de ensino, pesquisa e extensão, através do desenvolvimento de projetos que viabilizem a transformação da realidade local. Este relato de experiência descreve a vivência de um grupo de discentes do Programa de Pós-Graduação em Odontologia da Universidade Federal de Sergipe (UFS) durante o desenvolvimento e execução de um projeto de extensão universitária intitulada "Multiplicando Saberes: Ampliando os Cuidados em Saúde Bucal".

\section{MATERIAL E MÉTODO}

\section{Contexto}

O projeto de extensão "Multiplicando Saberes: Ampliando Cuidados em Saúde Bucal" está vinculado a um projeto de pesquisa intitulado "Impacto da Saúde Bucal na Qualidade de Vida de Crianças e Adolescentes com Deficiência”, desenvolvido dentro do Programa de Pós-Graduação em Odontologia (PRODONTO) da UFS e incorporado junto ao Núcleo de Popularização do Conhecimento Científico em Odontologia (NPCCO). O NPCCO, órgão de extensão voltado 
para atividades de inserção social do PRODONTO/UFS, tem por principal objetivo transpor o conhecimento científico em Odontologia produzido na Universidade para a sociedade, tomando seu papel na responsabilidade social do conhecimento e conquista da cidadania. Além disso, o NPCCO integraliza as atividades de pesquisa desenvolvidas no PRODONTO/UFS a ações de extensão, promovendo Educação em Saúde para as comunidades assistidas.

\section{Proposta}

O projeto de extensão "Multiplicando Saberes: Ampliando Cuidados em Saúde Bucal”, dentro da proposta de desenvolvimento de práticas educativas e promoção de saúde, visa implementar melhorias na qualidade de saúde bucal de crianças e adolescentes com deficiência, fornecendo informações aos cuidadores no que se refere aos cuidados com a saúde bucal e estimulando o autocuidado de maneira lúdica com a participação dos cuidadores.

\section{Público-alvo e Início das atividades}

O presente projeto de extensão possui como público-alvo crianças e adolescentes com deficiência, matriculados em escolas públicas e privadas que trabalham com educação inclusiva, crianças e adolescentes atendidos no Terceiro Setor (composto por entidades privadas da sociedade civil, que prestam atividade de interesse social, por iniciativa privada, sem fins lucrativos) ou Centros de Especialidade Odontológica (CEO), cuidadores e profissionais de educação que trabalham com todos os alunos participantes do projeto. Teve suas atividades iniciadas em dezembro de 2015, a partir da necessidade de promover educação em saúde bucal para essa população.

\section{Ações de extensão}

As ações extensionistas realizadas por meio desse projeto envolveram atividades educativas voltadas à cidadania e promoção de saúde bucal, oferecendo orientação e avaliação odontológica a crianças e adolescentes com deficiência e seus cuidadores, professores e orientadores educacionais, ampliando os cenários de prática no ensino da área da saúde para além dos muros da Universidade.

\section{RESULTADOS}

Um total de 162 crianças e adolescentes com deficiência foram incorporados no projeto das quais (com avaliação médica presente em seus cadastros, nas instituições onde se desenvolveu a pesquisa) 22 foram diagnosticadas com paralisia cerebral, 36 com transtorno do espectro autista, 13 com síndrome de Down e 91 com retardo mental. Quarenta e quatro cuidadores, a maioria do gênero feminino (92\%), também foram inseridos. A maioria dos cuidadores estão na faixa etária de 30 a 40 anos (54.5\%), são casados (38.6\%), têm de 2 a 3 filhos (63.6\%), têm até o ensino médio completo $(72.7 \%)$ e se dedicam integralmente ao cuidado das crianças ou adolescentes com deficiência (79.5\%). Cerca de 68,2\% não possuem renda formal. Dos 4 educadores inseridos no projeto, 3 são do gênero feminino e se dedicam parcialmente às atividades com crianças e 
adolescentes com deficiência. Todos os educadores apresentam ensino médio completo.

As atividades foram realizadas em 6 instituições: CEOs dos municípios de Nossa Senhora do Socorro e Aracaju, Escola de Educação Especial João Cardoso do Nascimento Júnior (Aracaju), Associação de Pais e Amigos dos Excepcionais (APAE) de Nossa Senhora do Socorro e Itabaiana e Centro de Integração Raio de Sol (Aracaju).

As ações de promoção de saúde foram desenvolvidas por meio de atividades educativas com metodologias ativas específicas para a população-alvo, buscando informá-los, conscientizá-los e motivá-los a realizarem o autocuidado em saúde tanto geral quanto bucal. As atividades foram organizadas da seguinte forma:

Palestras - O primeiro contato com os educadores, alunos e cuidadores se deu através de uma palestra educativa, na qual foi esclarecido o funcionamento do projeto e seus objetivos. As temáticas abordadas nas palestras, utilizando linguagem adequada ao público, envolveram a importância da saúde bucal relacionada com a saúde geral, escovação e o correto uso do fio dental, dieta alimentar $\mathrm{X}$ cárie dentária, formação de placa bacteriana, uso do flúor, prevenção de hábitos bucais deletérios (uso de chupeta, succção digital e onicofagia), e prevenção e conduta em casos de traumatismo dentário;

Atividades lúdicas - Cartilhas educativas abordando temas relativos à cárie dentária, doença periodontal, cuidados com alimentação, além de demonstração com macromodelo odontológico de técnicas de escovação, bem como adaptações das técnicas convencionadas, tendo em vista a participação dos cuidadores dos alunos com deficiência;

Atividades práticas com alunos, professores e cuidadores - A partir da orientação dada nas etapas anteriores, os participantes foram acompanhados durante a realização de higiene, sendo permitido o esclarecimento de dúvidas sobre os cuidados com a saúde bucal. Foi escolhido um local iluminado e conveniente. Para o desenvolvimento dessa atividade foi levada em consideração a capacidade de cooperação da criança e do adolescente para efetivação junto ao seu cuidador da atividade proposta;

Debates - Ações envolvendo todos os participantes nas diferentes instituições, avaliando o aspecto teórico e prático do que fora abordado, bem como os resultados obtidos durante o período.

Com a realização destas atividades de extensão, diversos resultados positivos foram percebidos, incluindo:

(1) a ampliação do vínculo entre discentes do programa e as crianças e adolescentes com deficiência, bem como seus cuidadores e educadores, por meio da troca de experiências e informações; 
(2) o desenvolvimento, por parte dos acadêmicos, de técnicas de comunicação e linguagem com o público-alvo, a capacidade de operacionalização de grupos, a obtenção de conhecimento técnico sobre os principais problemas bucais que acometem as pessoas com paralisia cerebral, retardo mental, autismo e síndrome de Down, e a aprendizagem sobre a vivência e dificuldades no cuidado com estas pessoas;

(3) a importância da educação em saúde como forma de inclusão social;

(4) o desenvolvimento de habilidades e competências por parte das crianças e adolescentes com deficiência, tais como hábitos de higiene, valores e socialização, promovendo melhoria na qualidade de vida dessas pessoas;

(5) a percepção da responsabilidade social da pós-graduação como importante agente de transformação da realidade.

\section{DISCUSSÃO}

O projeto de extensão "Multiplicando Saberes: Ampliando Cuidados em Saúde Bucal" associado ao projeto de pesquisa "Impacto da Saúde Bucal na Qualidade de Vida de Crianças e Adolescentes com Deficiência" leva informações aos cuidadores destes pacientes, evidenciando como a saúde bucal afeta a qualidade de vida. Estes necessitam de um acompanhamento intenso por parte dos seus cuidadores, os quais estão intimamente envolvidos no cuidado, suprindo as necessidades básicas de vida diária, a maior parte do dia, a fim de que aqueles possam ter a melhor qualidade de vida possível ${ }^{4}$.

De acordo com Barbosa et $\mathrm{al}^{5}$, a qualidade de vida relacionada à saúde é um importante componente auxiliar aos indicadores clínicos na avaliação da saúde do paciente. Diante da evidente relação que existe entre saúde bucal e qualidade de vida, é nítida a importância que os cuidadores exercem na vida destes pacientes. Aqueles necessitam receber informações e apoio por parte dos profissionais da saúde, para que os seus cuidados sejam sempre efetivos na manutenção da saúde geral e bucal destes pacientes.

Tendo em vista esse fato, evidencia-se que o projeto de extensão caracteriza-se como um elemento que preenche essa lacuna de transferência de conhecimentos realizando uma intervenção de forma continuada em determinada comunidade, e executa suas ações numa simbiose entre o acadêmico e o fazer/saber popular ${ }^{6}$.

A aproximação do acadêmico com os cuidadores através da extensão na pós-graduação traz benefícios tanto aos estudantes como a comunidade, a qual recebe informações que a ajudam a ter uma melhor qualidade de vida. O estudante torna-se um multiplicador de informações, e essa experiência fornece vivência e segurança ao acadêmico ${ }^{7,8}$. 
Este projeto de extensão proporcionou aos acadêmicos além da ampliação dos seus conhecimentos científicos sobre problemas bucais de pessoas com deficiência, a experiência de como enfrentar dificuldades no cuidado e na abordagem a estes pacientes. Assim como salientam os trabalhos de Fadel et al ${ }^{1}$ e de Pereira et al ${ }^{8}$ que relatam a importância da extensão para a formação do acadêmico.

Através da extensão, vários setores da sociedade se vinculam com a Universidade, onde a mesma oferece à comunidade conhecimentos e assistência, e recebe de volta informações essenciais sobre valores e cultura que serão replicados de forma válida e globalizados para toda a comunidade.

Apesar de ser notório que a extensão universitária e o trabalho voluntário trazem benefícios como a humanização do profissional da odontologia, auxiliam a formação técnico-científica e melhoram a saúde bucal da população mais carente, os mesmos ainda são insuficientes nas universidades brasileiras. As universidades que os inserem formam profissionais mais capacitados para enfrentar o mercado de trabalho, uma vez que as pessoas com deficiências estão tendo uma expectativa de vida cada vez maior, o que demanda também profissionais mais embasados para atendê-los, ressaltando-se, ainda, que estes pacientes são mais propensos a problemas bucais por todas as suas limitações ${ }^{9,10}$.

A Universidade perante a sociedade deve tornar possível a realização de uma reflexão que leve, através de uma análise crítica e cientificamente embasada, a sua transformação, bem como possibilitar a materialização de noções básicas de saúde e incentivar o autocuidado, e a capacitação de cuidadores de pessoas com deficiência, sendo um das principais desafios dos programas de extensão ${ }^{8}$.

\section{CONCLUSÃO}

Podemos observar que ocorre uma transferência de conhecimento, a qual leva da Universidade à comunidade informações sobre uma boa higiene bucal, e como a mesma melhora a qualidade de vida dos sujeitos. Estes são então elevados à condição de agentes ativos e multiplicadores das ações de promoção de saúde em nível individual e coletivo.

\section{REFERÊNCIAS}

1.Fadel CB, Bordin D, Kuhn E, Martins LD. O impacto da extensão universitária sobre a formação acadêmica em Odontologia. Interface (Botucatu) 2013.17:937-946. doi: $10.1590 / 180757622013.3811$

2.Moita FMG da SC, Andrade FCB de. Ensino-pesquisa-extensão: um exercício de indissociabilidade na pós-graduação. Rev Bras Educ 2009.14:269-280.

3.Boufleuer JP. Inserção social como quesito de avaliação da pós-graduação. Rev Educ Pública 2009. 18:371-382.

ISSN 1982-8829 Tempus, actas de saúde colet, Brasília, 13(3), 183-190, set, 2019. Epub Ju1/2020 
4. Curvero MM. Qualidade de vida em cuidadores de crianças e adolescentes com autismo. 73p. Tese (Mestrado em Ciências da Saúde). Universidade Federal de Uberlândia. Uberlândia. 2008.

5.Barbosa TS, Mialhe FL, Castilho ARF, et al. Qualidade de vida e saúde bucal em crianças e adolescentes: aspectos conceituais e metodológicos. Physis [online]. 2010; 20(1): 283-300.

6.Castilho LS de, Resende VLS, Pacheco, AR, Nunes, L, Lages FS .A formação do estudante de odontologia e educação em saúde para a população:a experiência de um projeto de extensão universitária. Interfaces- Revista de extensão Belo horizonte 2014.v.2, n.2, p.120-130. jan-jun

7.Nunes LSR, Gomes GM, Júnior LGS, Santos MCS, Miranda RS, Evangelista LM. Teia de aranha:uma visão crítica em saúde bucal. Revista eletrônica Gestão e Saúde, vol 6, suplemento 1, março 2015, p.793-99.

8.Pereira, V. Oficina de Saúde Bucal Aplicada na Operação Porta do Sol, Itatuba/PB e na E.E. Júlio de Mesquita Filho, São Paulo-SP. R. Eletr. de Extensão, Florianópolis, v. 13, n. 21, p.214$223,2016$.

9.Pereira LM, Mardero E, Ferreira SH, Kramer, PF, Cogo RB. Atenção odontológica em pacientes com deficiências: a experiência do curso de Odontologia da ULBRAS Canoas/RS. Stoamtos, v.16,n.31, jul/dez, 2010.

10.Pereira SM, Mialhe FL, Pereira LJ, Soares MF, Tagliaferro EPS et al. Extensão Universitária e Trabalho Voluntário na Formação do Acadêmico em Odontologia. Arq Odontol, Belo Horizonte, 47(2): 95-103, abr/jun 2011

Artigo apresentado em outubro 2019 Artigo aprovado em janeiro de 2020 Artigo publicado em julho de 2020 